\title{
Adherens Junctions, Desmosomes and Tight Junctions in Epidermal Barrier Function
}

\author{
Johanna M. Brandner ${ }^{1, \S}$, Marek Haftek ${ }^{*, 2, \S}$ and Carien M. Niessen ${ }^{3, \S}$ \\ ${ }^{I}$ Department of Dermatology and Venerology, University Hospital Hamburg-Eppendorf, Hamburg, Germany \\ ${ }^{2}$ University of Lyon, EA4169 Normal and Pathological Functions of Skin Barrier, E. Herriot Hospital, Lyon, France \\ ${ }^{3}$ Department of Dermatology, Center for Molecular Medicine, Cologne Excellence Cluster on Cellular Stress Responses \\ in Aging-Associated Diseases (CECAD), University of Cologne, Germany
}

\begin{abstract}
The skin is an indispensable barrier which protects the body from the uncontrolled loss of water and solutes as well as from chemical and physical assaults and the invasion of pathogens. In recent years several studies have suggested an important role of intercellular junctions for the barrier function of the epidermis. In this review we summarize our knowledge of the impact of adherens junctions, (corneo)-desmosomes and tight junctions on barrier function of the skin.
\end{abstract}

Keywords: Cadherins, catenins, claudins, cell polarity, stratum corneum, skin diseases.

\section{INTRODUCTION}

The stratifying epidermis of the skin physically separates the organism from its environment and serves as its first line of structural and functional defense against dehydration, chemical substances, physical insults and micro-organisms. The living cell layers of the epidermis are crucial in the formation and maintenance of the barrier on two different levels. First, keratinocytes ultimately form the outermost protective dead layer of the skin, the cornified layer, through a complex spatial and temporal differentiation process. Impairment of this differentiation results in a reduced stratum corneum (SC) barrier function, as can be seen e.g. in atopic dermatitis [1].

Second, the living cell layers themselves form a barrier by providing tight mechanical cohesion between the cells of the same and different epidermal layers. To establish this barrier the viable cells have to connect to each other by intercellular junctions that link intercellular contacts to the cytoskeleton, such as tight junctions, (corneo) desmosomes and adherens junctions (Fig. 1). Important examples for the indispensability of the viable cell layers are the severe outcomes of blistering skin diseases, e.g. epidermolyis bullosa simplex and pemphigus vulgaris, the first affecting the integrity of the cells, the latter the integrity of cell-cell junctions. In the last decades evidence emerged that the intercellular junctions not only directly contribute to the viable epidermal barrier but also have important contributions to the correct formation and maintenance of the stratum corneum barrier. This review will focus on the role of adherens junctions, (corneo)-desmosomes and tight junctions in barrier function of mammalian skin.

*Address correspondence to this author at the University of Lyon, EA4169 Normal and pathological functions of skin barrier, E. Herriot Hospital, Lyon, France; Tel: +33 472110292; Fax: 33 472110290;

E-mail: haftek@univ-lyon1.fr

${ }^{\S}$ All authors contributed equally to the manuscript, are corresponding authors and are named alphabetically

\section{ADHERENS JUNCTIONS}

Adherens junctions are intercellular structures that couple intercellular adhesion to the cytoskeleton thereby creating a transcellular network that coordinate the behavior of a population of cells. Adherens junctions are dynamic entities and also function as signal platforms that regulate cytoskeletal dynamics and cell polarity. As such, they regulate a diverse range of other cellular processes next to adhesion, such as cell shape, division, growth, apoptosis and barrier function. The molecular basis of adherens junctions is formed by two cell adhesion receptor complexes, the classical cadherin/catenin complex and the nectin/afadin complex, which both can link to the actin cytoskeleton. Classical cadherins are single transmembrane $\mathrm{Ca} 2+-$ dependent cell adhesion molecules that at their cytoplasmic face interact with catenins: P120ctn regulates cadherin cell surface stability and potentially connects adhesion to regulation of Rho GTPases whereas $\beta$-catenin links the cadherin to the actin regulator $\alpha$-catenin [2]. Nectins are IgG like adhesion molecules that bind the actin binding protein afadin.

Two types of classical cadherins are expressed in the epidermis: P-cadherin (cadherin 3), expressed in the basal layer mainly around and in hair follicles, and E-cadherin (cadherin 1) found in all layers of the epidermis (Fig. 2). It was recently reported that E-cadherin is a target of autoantibodies in pemphigus, a skin blistering disease [3]. Surprisingly, epidermal specific deletion of E-cadherin in the mouse did result in hair loss but did not cause any blistering, as was perhaps to be expected for an adhesion molecule like E-cadherin $[4,5]$. In contrast to what was implicated by several in vitro studies, ultrastructural normal desmosomes were present. However, the skin of epidermal E-cadherin knockout mice showed extensive water loss resulting in perinatal death [6]. Closer analysis revealed a loss of insideout but not outside in barrier function that was associated with impaired in vivo and in vitro tight junction function [6]. 


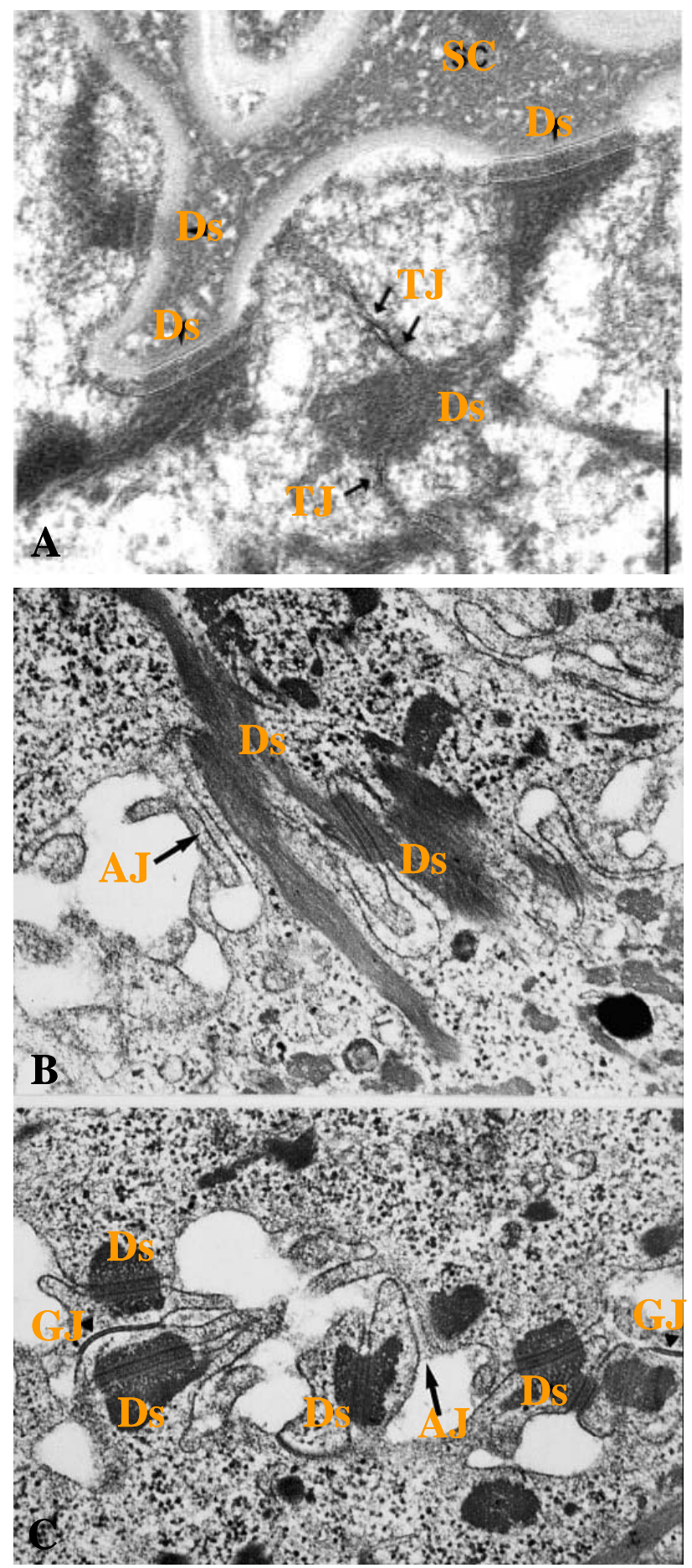

Fig. (1). Close spatial relationship between adherens junctions (AJ), desmosomes (Ds) and tight junctions (TJ) in human epidermis, as seen with electron microscopy. (A): fetal, (B, C) adult human skin. The micrograph of TJ (small arrows pointing to the TJ "kissing points") has been taken from [30]. GJ = gap junctions. $\mathrm{SC}=$ stratum corneum. Bar $=500 \mathrm{~nm}$.

Loss of P-cadherin in mice did not reveal any obvious phenotypes [7] even though mutations in human P-cadherin are associated with a hair disorder, hypotrichosis with juvenile macular dystrophy (OMIM \# 601553) [8], and with ectodermal dysplasia associated with ectrodactyly and macular dystrophy (EEM; OMIM \# 225280) [9]. These results thus suggest that $\mathrm{E}$ - and P-cadherin either serve partially differential functions or have a different functional overlap in human and mouse.

Epidermal inactivation in mice revealed overlapping and specific functions for the cadherin associated catenins. Loss of $\beta$-catenin in the epidermis confirmed its importance in the Wnt signaling pathway and its role in hair follicle morphogenesis and stem cell regulation. However, no obvious defects in intercellular adhesion and junction formation were observed, most likely because plakoglobin substituted for $\beta$-catenin in the cadherin complex. Inactivation of p120ctn in the epidermis resulted in reduced adherens junctions and skin inflammation associated with activation of $\mathrm{Nf \kappa B}$ [10]. An almost complete loss of adherens junctions, reduced desmosomes and subsequent skin blistering was observed when $\alpha$-catenin was deleted from the epidermis [11]. Since both P-cadherin and Ecadherin interact with the catenins, loss of a single cadherin may be insufficient to phenocopy deletion of one of the catenins. This is in agreement with studies in human keratinocytes. These show that a combination of antibodies to E- and P-cadherin inhibited not only the formation of adherens junctions but also desmosomes and interfered with stratification. Recent in vitro and in vivo studies using knockout/knockdown strategies confirmed that classical cadherins are crucial not only for the formation of tight junctions but also regulate desmosome formation $[12,13]$. This does not depend on a specific function of E- and Pcadherin but is related to the relative total expression levels of these two classical cadherins together.

\section{DESMOSOMES - CORNEODESMOSOMES}

Desmosomes are "mechanical" junctions, involved primarily in cell cohesion [14]. They are composed of the desmosomal cadherins, which, similar to the classical cadherins of adherens junctions, are part of the cadherin superfamily. Desmogleins 1-4 and desmocollins 1-3 are found in the human epidermis (Fig. 2). The intracellular ends of desmosomal cadherins are inserted in the molecular network of adaptor proteins forming desmosomal plaques, to which keratin filaments bind. Two families of plaque proteins can be distinguished in desmosomes: i) plakoglobin and plakophilins, which belong to the armadillo repeat protein family that also includes $\beta$-catenin and p120ctn, and ii) desmoplakin, envoplakin, periplakin and plectin from the plakin family. As keratinocytes move through the epidermal layers, they constantly form and retrieve desmosomes at the cell periphery. During this turnover, the molecules that compose junctions (even without physical dissociation of the structure) are also constantly replaced. According to the level of keratinocyte differentiation, desmogleins 2 and 3 from the lower epidermal compartment are progressively substituted by desmogleins 1 and 4 in the upper viable epidermal layers. In the same way desmocollin 3 is replaced by desmocollin 1 (Fig. 2). This differentiation -dependent composition of desmosomes coincides with the increase of their mechanical stability. The fact that extracellular calcium levels are elevated in the upper epidermis certainly also contributes to the desmosome stabilization. In the basal layer, desmosomes are infrequent and small; their size and number rise significantly in the spinous layers and decrease subsequently in the granular compartment [15]. A host of dermatoses is 


\section{Distribution of cadherins in human epidermis}

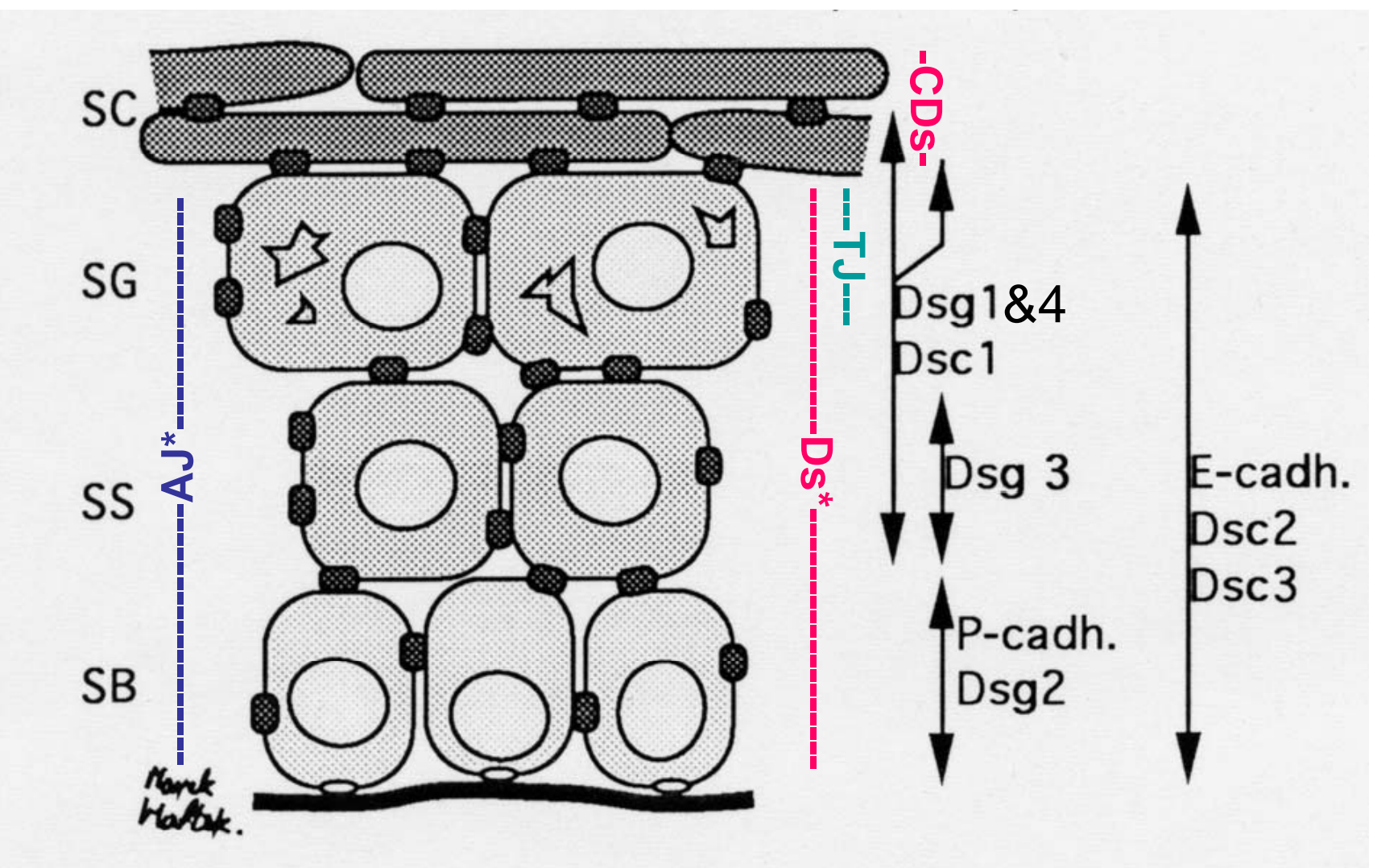

Fig. (2). Schematic drawing of the localization of classical and desmosomal cadherins as well as the corresponding junctions (black dots) in human epidermis. AJ = adherens junctions, $\mathrm{Ds}=$ desmosomes and $\mathrm{CDs}=$ corneodesmosomes. $*$ Composition of these junctions varies in different epidermal layers. SC: stratum corneum, SG: stratum granulosum, SS: stratum spinosum, SB: stratum basale.

related to desmosome dysfunction. In several autoimmune diseases autoantibodies are directed against various desmosome components, e.g., desmoglein 3 in pemphigus vulgaris, desmoglein 1 in pemphigus foliaceus, or several plakins in paraneoplastc pemphigus. Hereditary skin diseases provoked by mutations in genes coding for desmosomal proteins, like plakophilin 1 which causes an autosomal recessive skin fragility - ectodermal dysplasia syndrome with skin, hair and nail defects, or desmoplakin and desmoglein 1 that underlie the autosomal dominant striate palmoplantar keratoderma (OMIM \# 601975, 125647, and 125670, respectively), provide further insights to functions of various components of these junctions. Plakoglobin has been shown to be defective in Naxos disease (OMIM \# 173325), which results in a cardiomyopathy and growth of abnormal hair, whereas desmosome regulation through intracellular calcium levels has been revealed by discovery of mutations in calcium pumps in Hailey-Hailey disease and Darier disease (OMIM \# 604384 and 108740) [16-18].

In the stratum granulosum and at the interface between the living epidermal layers and the stratum corneum, calcium activated transglutaminase 1 cross-links proteins present under and close to the keratinocyte plasma membrane. This includes transmembrane and intracellular portions of cell-cell junctions. Thus, desmosomes can no longer be recycled and, from that level on, their destiny is to be degraded by adequate hydrolases. In the horny layer, the morphology of desmosomes changes dramatically. The layered structure of the intercellular portion of the junction is lost, whereas the intracellular plaque becomes embedded within the cross-linked cornified envelope. Nevertheless, these corneodesmosomes (Fig. 2) remain functional and their ordinate digestion will eventually permit dissociation of the horny layer and desquamation of superficial corneocytes [19, 20].

In the lower SC, called the SC compactum, corneodesmosomes are frequent and evenly distributed on the corneocyte surface. In fact, this reflects normal distribution of desmosomes from the last living layers, the junctions being immobilized at the cell periphery by transglutaminase -mediated cross-linking. Degradation of these plug-like structures occurs first between the overlying strata of corneocytes resulting in their vertical separation and creation of the "basket-weave" pattern typical of the SC 
disjunctum. At this point, corneodesmosomes are located only at the overlapping edges of the corneocytes, in cell-cell interdigitation zones, and the flat corneocyte discs remain laterally attached with these junctions. When the lateral / peripheral corneodesmosomes disappear, in their turn, the corneocytes are gradually shed at the skin surface. The reasons for the preferential loss of corneodesmosomes from the relatively flat upper and lower faces of corneocytes may include: i) a higher impact of shearing forces on junctions between flat rigid surfaces, when applied to the skin or naturally occurring during changes of the corneocyte volume due to variations in the water content; ii) easier access for the enzymes, displaced by self-organizing lipid bilayers, when compared to the convoluted lateral intercorneocyte spaces; iii) an additional protection of the peripheral / lateral junctions by supplementary structures, like remnants of strategically placed tight junctions cross-linked to cornified envelopes at the periphery of corneocytes [21].

Desmoglein 1, desmocollin 1 and corneodesmosin have been shown to be structural components of the corneodesmosome cores. Synthesized and secreted by the granular layer keratinocytes, corneodesmosin is also an adhesive protein [22]. Its presence in the corneodesmosome plug, and the close association observed between corneodesmosin degradation at the epidermal surface and corneocyte shedding suggest that the protein plays a major role in reinforcing cell cohesion in the stratum corneum [23]. An alternative proposal, suggesting a possible protective role of cornedesmosin against premature proteolysis of the junction proteins has also been advanced but remains unverified [24]. Corneodesmosomal core and plaque proteins are covalently cross-linked to the other components of the cornified cell envelopes, thereby anchoring them in place [25].

Interplay of proteases and protease inhibitors regulated by $\mathrm{pH}$ is involved in orderly hydrolysis of the corneodesmosome plugs [26]. Deglycosylation of structural proteins largely accelerates this process [27]. Processing of the intercellular lipids with lipases and humidity also influence mobility of the enzymes within the largely hydrophobic extracellular spaces [28]. So far, only one hereditary syndrome has been linked to the deficiency in corneodesmosin expression, namely autosomal dominant hypotrichosis simplex. However, corneodesmosome persistence within the stratum corneum due to their defective processing can be related with several hyperkeratotic conditions like certain ichthyoses or seasonal/senile dry skin $[23,29]$.

\section{TIGHT JUNCTIONS}

Tight Junctions (TJ) are occluding junctions. In simple epithelial and endothelial cells they were shown to fulfil different functions, the most prominent being the paracellular "barrier function" and the "fence function", which separates the apical membrane domain from the basolateral part.

For keratinizing epithelia, it was originally thought that the formation of the stratum corneum barrier obviated the need for TJ. However, typical TJ structures, i.e. so-called "kissing points", have been identified in man and mouse in the lateral plasma membranes of the stratum granulosum
(SG; Figs. 2, 3) [30, 31]. In addition a large number of TJ proteins, including claudins (Cldns) and ZO-proteins which are known to be important for barrier function in simple epithelial cells, were found in this layer (Fig. 3) [32]. Interestingly, early ultrastructural electron dense tracer studies revealed the presence of a functional barrier in the SG $[33,34]$, but this was mainly attributed to the intercellular deposition of lamellar bodies [35].

The first functional evidence that epidermal barrier function required a tight junction component came from claudin-1 deficient mice, which die of massive trans epidermal water loss (TEWL) due to impaired barrier function of the SG [36]. However, it is not clear yet whether the increase of TEWL is due to an increased permeability of $\mathrm{TJ}$ for water or whether secondary changes in the SC due to the loss of TJ function are responsible for this dysfunction. A detailed investigation of the skin of these mice showed that a dermally injected 557 Da tracer is no longer stopped at TJ in the SG (marked by occludin) [31]. In addition, another mouse model with the phenotype "skin barrier defect", the epidermal E-cadherin knockout mouse, was shown by careful examination to be characterized by the loss of Cldn-1 in the SG and a miclocalization of further TJ proteins [6]. Thus it is now widely accepted that TJ and TJ proteins are critical for the barrier function of murine stratifying epithelia.

Much less is known about TJ function in human skin. As mentioned, TJ proteins as well as typical TJ structures were identified in human epidermis. The loss of Cldn-1 in the NISCH (neonatal ichthyosis, sclerosing cholangitis)syndrome (OMIM \# 607626) results in a severe skin phenotype which emphasises its importance also in human skin. However this disease is not lethal, which hints for compensatory mechanism in man which is not present in mice.

In cultured keratinocytes functional $\mathrm{TJ}$ which form a barrier for ions as well as for larger molecules were demonstrated in cells of murine and human origin [37-39]. It was shown that the atypical protein kinase $\mathrm{C}$ as well as the small GTPase Rac1 and the Rac-specific guanine exchange factor Tiam 1 are crucial for the formation of functional TJ in keratinocytes [37, 38].

Skin diseases which are characterized by impaired SC barrier function, e.g. psoriasis vulgaris, show an alteration of TJ protein expression and localization. Particularly, occludin and ZO-1 are up-regulated and found in more cell layers than in normal skin. On the other hand side, claudins are downregulated, at least in some psoriatic plaques [40-44]. On the ultrastructural level TJ structures were identified in the spinous cell layer of psoriatic skin [44]. This suggests that the epidermis attempts to rescue the stratum corneum defect through the formation of a functional TJ barrier in deeper layers of the epidermis that normally do not form a TJ barrier.

In addition to their presence in the SG most TJ proteins are also found in deeper layers of the epidermis. The mechanisms that restrict the assembly of a TJ structure to the uppermost living layers of stratifying epithelia are unclear (Fig. 3). Since many TJ components are expressed throughout the epidermal layers, it is possible that a local signal in 


\section{Localization of tight junction (TJ) proteins and TJ structures in human epidermis}

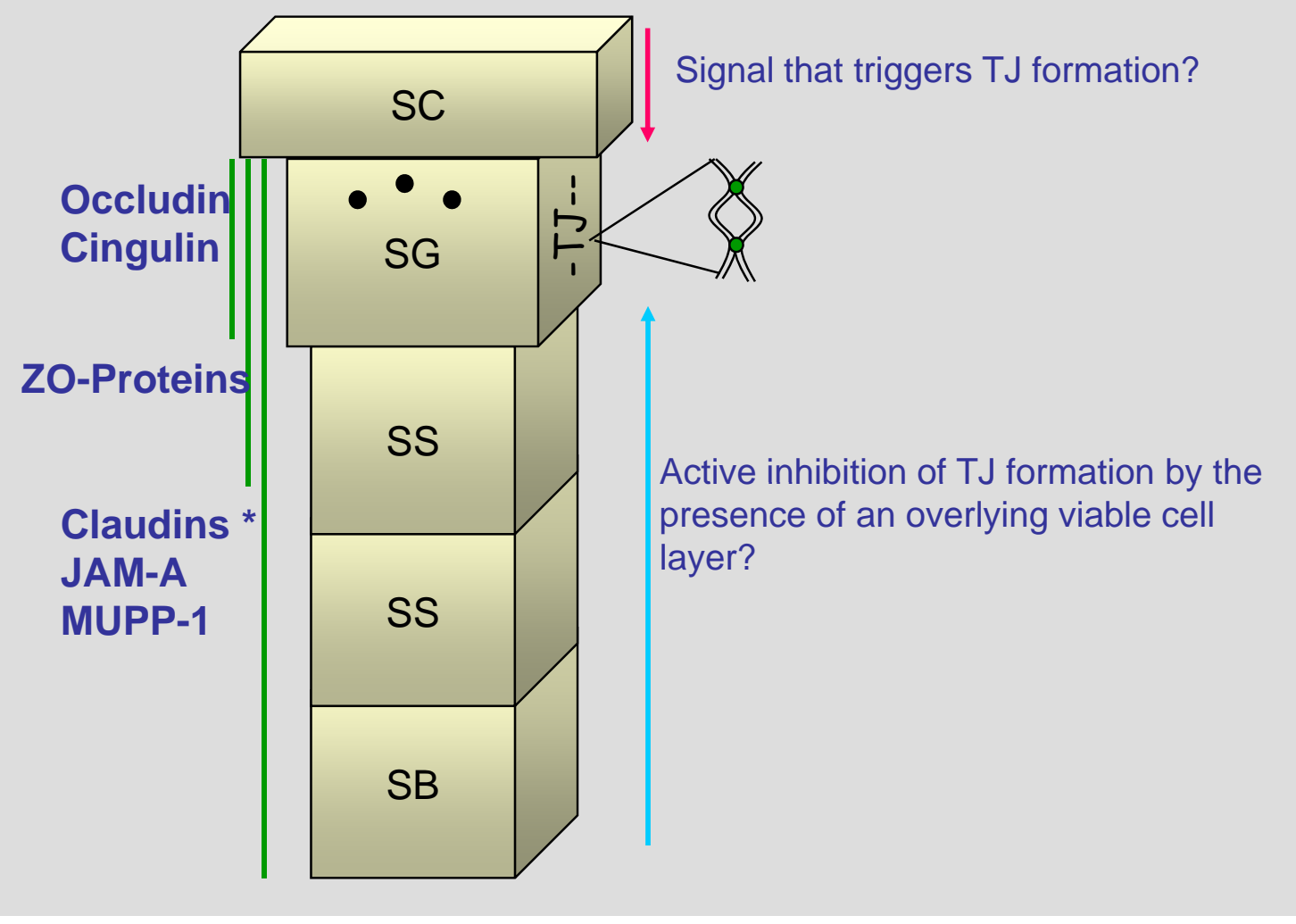

Fig. (3). Schematic drawing of the localization of tight junction (TJ) proteins and TJ structures in human epidermis. * different isoforms in different epidermal layers; SC: stratum corneum, SG: stratum granulosum, SS: stratum spinosum, SB: stratum basale.

the SG triggers TJ formation. We speculate that the presence of a competent horny layer may provide this signal, similar to other epithelia which secrete and are polarized by an apical matrix (e.g., such as follicular epithelia in flies that secrete an apical cuticle). Alternatively, TJ formation in the lower epidermal layers may be actively inhibited by the presence of an overlying viable cell layer. Either mechanism suggests that the restriction of TJ to the most superficial living layer in stratifying epidermis or apical region of simple epithelia may be conserved. Indeed, our data discussed here and elsewhere support this argument: Ecadherin is required for $\mathrm{TJ}$ formation in both simple and stratifying epithelia. Blocking E-cadherin in vitro inhibits TJ in simple epithelia [45] as does genetic loss of epidermal Ecadherin. Similarly, blocking aPKC inhibition interferes with TJ in both epithelia $[37,38,46]$. Thus, junctional and polarity proteins required in simple epithelia are also turning out to be critical for epidermal barrier function, suggesting these processes may be mechanistically related.

The epidermis is not a classically polarized epithelium like intestine; in which $\mathrm{TJ}$ separate the basolateral and apical membrane domains. Instead the epidermis establishes a form of tissue polarity across the different stratifying cell layers with the basal cells forming the basal side and the SG forming the viable apical boundary. Formation of the SC depends on the fusion of lamellar bodies with plasma membranes at the "apical" transition between the viable granular layer keratinocytes and the horny layer. It is therefore tempting to speculate that the restricted presence of functional TJ in the SG also relates to their "fence" function thereby regulating the targeted secretion of "apical" protein and lipid vesicles. This would imply that changes in TJ affect the SC barrier which could indeed be observed: Forced expression of claudin- 6 in the upper layers of the epidermis does not only affect $\mathrm{TJ}$ but also results in defects of the SC [47]. In addition, deficiency of the serine protease (CAP) $1 /$ Prss 8 in the epidermis also disturbs both barriers [48]. Although the underlying mechanisms are unknown, they may involve the coordinated regulation of both barriers by signal molecules such as IKK1. Inactivation of IKK1 in the epidermis severely impairs skin barrier function associated with improper epidermal lipid processing and changes in TJ component expression. Epidermal IKK1 function is independent of $\mathrm{Nf} \kappa \mathrm{B}$ signaling but regulates the expression of retinoic acid receptor target genes, many of which are involved in epidermal barrier function [49]. 


\section{CONCLUSION}

In summary, the data of recent years clearly show that the barrier function of the mammalian skin is a very complex system involving several components, e.g. the lipids and the corneocytes of the stratum corneum, the antimicrobial peptides and the intercellular junctions. Further elucidation of the contribution of the individual components to epidermal barrier function and their interactions in healthy and diseased skin are challenging tasks for the future.

\section{REFERENCES}

[1] O'Regan GM, Sandilands A, McLean WH, Irvine AD. Filaggrin in atopic dermatitis. J Allergy Clin Immunol 2009; 124: R2-6.

[2] Perez-Moreno M, Fuchs E. Catenins: keeping cells from getting their signals crossed. Dev Cell 2006; 11: 601-12.

[3] Evangelista F, Dasher DA, Diaz LA, Prisayanh PS, Li N. Ecadherin is an additional immunological target for pemphigus autoantibodies. J Invest Dermatol 2008; 128: 1710-8.

[4] Tinkle CL, Lechler T, Pasolli HA, Fuchs E. Conditional targeting of E-cadherin in skin: insights into hyperproliferative and degenerative responses. Proc Natl Acad Sci USA 2004; 101: 552-7.

[5] Young P, Boussadia O, Halfter $\mathrm{H}$, et al. E-cadherin controls adherens junctions in the epidermis and the renewal of hair follicles. EMBO J 2003; 22: 5723-33.

[6] Tunggal JA, Helfrich I, Schmitz A, et al. E-cadherin is essential for in vivo epidermal barrier function by regulating tight junctions. EMBO J 2005; 24: 1146-56.

[7] Radice GL, Ferreira-Cornwell MC, Robinson SD, et al. Precocious mammary gland development in P-cadherin-deficient mice. J Cell Biol 1997; 139: 1025-32.

[8] Sprecher E, Bergman R, Richard G, et al. Hypotrichosis with juvenile macular dystrophy is caused by a mutation in $\mathrm{CDH} 3$, encoding P-cadherin. Nat Genet 2001; 29: 134-6.

[9] Kjaer KW, Hansen L, Schwabe GC, et al. Distinct CDH3 mutations cause ectodermal dysplasia, ectrodactyly, macular dystrophy (EEM syndrome). J Med Genet 2005; 42: 292-8.

[10] Perez-Moreno M, Davis MA, Wong E, Pasolli HA, Reynolds AB, Fuchs E. p120-catenin mediates inflammatory responses in the skin. Cell 2006; 124: 631-44.

[11] Vasioukhin V, Bauer C, Degenstein L, Wise B, Fuchs E. Hyperproliferation and defects in epithelial polarity upon conditional ablation of alpha-catenin in skin. Cell 2001; 104: 60517 .

[12] Tinkle CL, Pasolli HA, Stokes N, Fuchs E. New insights into cadherin function in epidermal sheet formation and maintenance of tissue integrity. Proc Natl Acad Sci USA 2008; 105: 15405-10.

[13] Michels C, Buchta T, Bloch W, Krieg T, Niessen CM. Classical cadherins regulate desmosome formation. J Invest Dermatol 2009; 129: 2072-5.

[14] Kowalczyk AP, Bornslaeger EA, Norvell SM, Palka HL, Green KJ. Desmosomes: intercellular adhesive junctions specialized for attachment of intermediate filaments. Int Rev Cytol 1999; 185: 237-302.

[15] McMillan JR, Haftek M, Akiyama M, et al. Alterations in desmosome size and number coincide with the loss of keratinocyte cohesion in skin with homozygous and heterozygous defects in the desmosomal protein plakophilin 1. J Invest Dermatol 2003; 121: 96-103.

[16] Ayub M, Basit S, Jelani M, et al. A homozygous nonsense mutation in the human desmocollin-3 (DSC3) gene underlies hereditary hypotrichosis and recurrent skin vesicles. Am J Hum Genet 2009; 85: 515-20.

[17] Cirillo N, Prime SS. Desmosomal interactome in keratinocytes: a systems biology approach leading to an understanding of the pathogenesis of skin disease. Cell Mol Life Sci 2009; 66: 3517-33.

[18] Nguyen B, Dusek RL, Beaudry VG, Marinkovich MP, Attardi LD. Loss of the desmosomal protein perp enhances the phenotypic effects of pemphigus vulgaris autoantibodies. J Invest Dermatol 2009; 129: 1710-8.

[19] Egelrud T, Hofer PA, Lundstrom A. Proteolytic degradation of desmosomes in plantar stratum corneum leads to cell dissociation in vitro. Acta Derm Venereol 1988; 68: 93-7.
[20] Simon M, Jonca N, Guerrin M, et al. Refined characterization of corneodesmosin proteolysis during terminal differentiation of human epidermis and its relationship to desquamation. J Biol Chem 2001; 276: 20292-9.

[21] Haftek M, Callejon S, Sandjeu Y, et al. Tight junction-like structures contribute to the lateral contacts between corneocytes and protect corneodesmosomes from premature degradation. J Invest Dermatol 2009; 129: S65.

[22] Jonca N, Guerrin M, Hadjiolova K, et al. Corneodesmosin, a component of epidermal corneocyte desmosomes, displays homophilic adhesive properties. J Biol Chem 2002; 277: 5024-9.

[23] Haftek M, Simon M, Kanitakis J, et al. Expression of corneodesmosin in the granular layer and stratum corneum of normal and diseased epidermis. Br J Dermatol 1997; 137: 864-73.

[24] Lundstrom A, Serre G, Haftek M, Egelrud T. Evidence for a role of corneodesmosin, a protein which may serve to modify desmosomes during cornification, in stratum corneum cell cohesion and desquamation. Arch Dermatol Res 1994; 286: 369-75.

[25] Haftek M, Serre G, Mils V, Thivolet J. Immunocytochemical evidence for a possible role of cross-linked keratinocyte envelopes in stratum corneum cohesion. J Histochem Cytochem 1991; 39: 1531-8.

[26] Ovaere P, Lippens S, Vandenabeele P, Declercq W. The emerging roles of serine protease cascades in the epidermis. Trends Biochem Sci 2009; 34: 453-63.

[27] Walsh A, Chapman SJ. Sugars protect desmosome and corneosome glycoproteins from proteolysis. Arch Dermatol Res 1991; 283: 174-9.

[28] Haftek M, Teillon MH, Schmitt D. Stratum corneum, corneodesmosomes and ex vivo percutaneous penetration. Microsc Res Tech 1998; 43: 242-9.

[29] Leclerc EA, Huchenq A, Mattiuzzo NR, et al. Corneodesmosin gene ablation induces lethal skin-barrier disruption and hair-follicle degeneration related to desmosome dysfunction. J Cell Sci 2009; 122: 2699-709.

[30] Brandner JM, Kief S, Grund C, et al. Organization and formation of the tight junction system in human epidermis and cultured keratinocytes. Eur J Cell Biol 2002; 81: 253-63.

[31] Furuse M, Hata M, Furuse K, et al. Claudin-based tight junction are crucial for the mammalian epidermal barrier: a lesson from claudin-1-deficient mice. J Cell Biol 2002; 156: 1099-111.

[32] Brandner JM. Tight junctions and tight junction proteins in mammalian epidermis. Eur J Pharm Biopharm 2009; 72: 289-94.

[33] Elias PM, Friend DS. The permeability barrier in mammalian epidermis. J Cell Biol 1975; 65: 180-91.

[34] Logan KR, Hopwood D, Milne G. Cellular junctions in human oesophageal epithelium. J Pathol 1978; 126: 157-63.

[35] Elias PM, McNutt NS, Friend DS. Membrane alterations during cornification of mammalian squamous epithelia: a freeze-fracture, tracer, and thin-section study. Anat Rec 1977; 189: 577-94.

[36] Furuse M, Hata M, Furuse K, et al. Claudin-based tight junctions are crucial for the mammalian epidermal barrier: a lesson from claudin-1-deficient mice. J Cell Biol 2002; 156: 1099-111.

[37] Helfrich I, Schmitz A, Zigrino P, et al. Role of aPKC isoforms and their binding partners Par3 and Par6 in epidermal barrier formation. J Invest Dermatol 2007; 127: 782-91.

[38] Mertens AE, Rygiel TP, Olivo C, van der Kammen R, Collard JG. The Rac activator Tiam1 controls tight junction biogenesis in keratinocytes through binding to and activation of the Par polarity complex. J Cell Biol 2005; 170: 1029-37.

[39] Yuki T, Haratake A, Koishikawa H, Morita K, Miyachi Y, Inoue S Tight junction proteins in keratinocytes: localization and contribution to barrier function. Exp Dermatol 2007; 16: 324-30.

[40] Brandner JM, Kief S, Wladykowski E, Houdek P, Moll I. Tight junction proteins in the skin. Skin Pharmacol Physiol 2006; 19: 717.

[41] Kirschner N, Poetzl C, von den Driesch P, et al. Alteration of tight junction proteins is an early event in psoriasis: putative involvement of proinflammatory cytokines. Am J Pathol 2009; 175: 1095-106.

[42] Pummi K, Malminen M, Aho H, Karvonen S-L, Peltonen J, Peltonen S. Epidermal tight junctions: ZO-1 and occludin are expressed in mature, developing, and affected skin and in vitro differentiating keratinocytes. J Invest Dermatol 2001; 117: 105058 
[43] Watson RE, Poddar R, Walker JM, et al. Altered claudin expression is a feature of chronic plaque psoriasis. J Pathol 2007; 212: 450-8.

[44] Yoshida Y, Morita K, Mizoguchi A, Ide C, Miyachi Y. Altered expression of occludin and tight junction formation in psoriasis. Arch Dermatol Res 2001; 293: 239-44.

[45] Gumbiner B, Stevenson B, Grimaldi A. The role of the cell adhesion molecule uvomorulin in the formation and maintenance of the epithelial junctional complex. J Cell Biol 1988; 107: 1575-87.

[46] Suzuki A, Yamanaka T, Hirose T, et al. Atypical protein kinase C is involved in the evolutionarily conserved par protein complex and plays a critical role in establishing epithelia-specific junctional structures. J Cell Biol 2001; 152: 1183-96.

[47] Turksen K, Troy TC. Permeability barrier dysfunction in transgenic mice overexpressing claudin 6. Development 2002; 129: 1775-84.

[48] Leyvraz C, Charles RP, Rubera I, et al. The epidermal barrier function is dependent on the serine protease CAP1/Prss8. J Cell Biol 2005; 170: 487-96.

[49] Gareus R, Huth M, Breiden B, et al. Normal epidermal differentiation but impaired skin-barrier formation upon keratinocyte-restricted IKK1 ablation. Nat Cell Biol 2007; 9: 4619.

(C) Brandner et al.; Licensee Bentham Open.

This is an open access article licensed under the terms of the Creative Commons Attribution Non-Commercial License (http://creativecommons.org/licenses/by-nc/ 3.0/) which permits unrestricted, non-commercial use, distribution and reproduction in any medium, provided the work is properly cited. 